\title{
Perturbation or Function? Intrinsic brain dynamics in the Default Mode Network predict involuntary fluctuations of visual awareness
}

Dian Lu

University of Cambridge

Emmanuel Stamatakis ( $\square$ eas46@cam.ac.uk)

University of Cambridge https://orcid.org/0000-0001-6955-9601

Shruti Naik

Cognitive Nueuroimaging Unit, INSERM, CEA, Universite' Paris-Saclay

David Menon

University of Cambridge https://orcid.org/0000-0002-3228-9692

\section{Article}

Keywords: cognition, brain activity, consciousness

Posted Date: July 13th, 2021

DOI: https://doi.org/10.21203/rs.3.rs-646785/v1

License: (9) This work is licensed under a Creative Commons Attribution 4.0 International License.

Read Full License

Additional Declarations: There is NO Competing Interest.

Version of Record: A version of this preprint was published at Nature Communications on November 14th, 2022. See the published version at https://doi.org/10.1038/s41467-022-34410-6. 


\title{
Perturbation or Function? Intrinsic brain dynamics in the Default Mode Network predict involuntary fluctuations of visual awareness
}

\author{
Dian Lyu ${ }^{1,2}$, Shruti Naik ${ }^{4}$, David K. Menon ${ }^{1,3}$, Emmanuel A. Stamatakis ${ }^{1,2 *}$ \\ ${ }^{1}$ University Division of Anaesthesia, University of Cambridge, Addenbrooke's Hospital, \\ Hills Rd, CB2 0SP Cambridge, UK \\ ${ }^{2}$ Department of Clinical Neuroscience, University of Cambridge, Addenbrooke's \\ Hospital, Hills Rd, CB2 0SP Cambridge, UK \\ ${ }^{3}$ Wolfson Brain Imaging Centre, University of Cambridge, Cambridge Biomedical \\ Campus (Box 65), CB2 0QQ, Cambridge, UK \\ ${ }^{4}$ Cognitive Nueuroimaging Unit, INSERM, CEA, Universite' Paris-Saclay, NeuroSpin \\ centre, 91191 Gif/Yvette, France
}

${ }^{*}$ To whom correspondence should be addressed; E-mail: eas46@cam.ac.uk

\begin{abstract}
Brain activity is intrinsically organised into spatiotemporal patterns, but it is still not clear whether the intrinsic dynamical patterns are functional or epiphenomenal. Using a simultaneous fMRI-EEG implementation of a well-known bi-stable visual task, we showed that the latent transient states $(\sim 100$ ms) in the intrinsic EEG oscillations can predict the upcoming involuntarily perceptual transitions. The critical state predicting a dominant perceptual transition was characterised by the phase coupling between the precuneus (PCU), a key node of the Default Mode Network (DMN), and the primary visual cortex (V1). The interaction between the lifetime of this state and the PCU->V1 causal effect is correlated with the rate of perceptual fluctuation. Our study suggests that the brain's endogenous dynamics are phenomenologically relevant, as they can trigger a diversion between potential visual processing pathways, while external stimuli remain the same. In this sense, the intrinsic DMN dynamics pre-empt the content of consciousness.
\end{abstract}




\section{Introduction}

Being aware is believed to be a globally "illuminated" inner state when locally encoded information gets propagated through downstream pathways and becomes accessible to other processing streams in the brain (1). However, the mechanism of the propagation process is largely unknown. Recent theoretical developments in brain dynamics suggest that the spontaneous information propagation may be empowered by the intrinsic ignition of neural activity (2). Empirically, intrinsic brain activity has been extensively studied during resting state when no external stimuli are presented to participants. At rest, neural activity from certain distant regions is correlated forming what are known as large-scale networks (3). We know that external perturbations can interrupt or bias the global cascades of information propagation (e.g. visual masking and priming). One untested hypothesis is that this process can also be influenced by intrinsic perturbations that originate from "background" dynamics of largescale brain networks (2). To test this hypothesis, we used a simultaneous fMRI-EEG implementation of the well-known Binocular rivalry (BR) paradigm to investigate whether and how intrinsic brain activity of a large-scale brain network influences the involuntary perceptual fluctuations during bistable visual awareness.

$\mathrm{BR}$ is a robust visual phenomenon where the participant perceives continuously alternating images even though the visual stimuli presented to the viewer, remain the same. To successfully trigger this phenomenon, dissimilar images need to be presented to the two eyes (one eye's view is blocked from the other); and to ensure balanced representations of the two images (or "percepts" as we shall call them in this paper), the two images should be distinguishable but of comparable cognitive load. Behavioural studies have established that the alternation of the two percepts is stochastic and beyond the participant's volitional control, but it is not random: duration of each percept follows a log-normal probability distribution (4) and on average differs notably from person to person (5). BR has been a popular paradigm for researchers aiming to study visual awareness as well as the neural correlates of consciousness (NCCs) (6-8). The classical model for explaining this phenomenon is called the (general) mutual inhibition model (MIM), which takes the form of a non-linear dynamic system (9). Although the model can have many variations in terms of the configurations of the local neural circuitry for inducing the counterbalancing mutual inhibition (9), it generally requires mutually inhibitory neurons encoding the representations of the two images (which exist as attractors in the model), and 
possible sources of perturbations, i.e., global and/or local adaptation of the neuronal populations as well as neuronal noise (10-13). A dominant percept during BR can be considered as a stable state/equilibrium achieved by the neuronal activity settling in one of the two possible attractors (13), while the state can escape from the current attractor under the influence of noise or habituation processes (such as spike frequency adaptation and synaptic fatigue) $(9,11,13)$. The MIM has been successful in fitting behavioural data measured during experiments, but empirical neural evidence to support the model is still lacking (14). In fact, this explanatory framework only focuses on the dynamics of the local neural circuitry, but totally ignores the background neural dynamics. However, the intrinsic dynamics of brain networks may have a lot of interactions with, and by which modulate, the activity of the local sensory region $(1,15)$.

Intrinsic brain activity is not random, instead, it is spatiotemporally organised into specific reproducible states (16-19). Although the spatial patterns of large-scale networks are mostly derived from unconstrained resting state, they can be identified across different tasks as well, possibly serving as a scaffold for various brain processes $(3,20)$. One of the large-scale networks that has achieved a prominent position in recent literature is the default mode network (DMN) (21). The DMN encompasses a wide range of associative regions across the prefrontal, temporal and parietal lobes, and consumes a major part of the brain's energy budget (22). A functional gradient analysis suggested that the DMN is situated at the top of the brain's information processing hierarchy, where multi-modal sensory information is integrated and highly abstract information (such as a concept of "self") is formed (23). Dynamic neural state studies using Hidden Markov models have shown that DMN activity dominates the latent brain states which are characterised by the synchronisation of high-order cognitive networks; where latent states are defined as recurring transient states that are assumed to drive the observed global neural dynamics $(16,19)$. Using control theory, it was also suggested that the DMN regions have the highest capability of steering the whole brain from one state to another, given the structural architecture of the brain (24).

Clinical studies have already highlighted the significance of the DMN in consciousness (2527), but little work has been done to investigate the role of the DMN in visual awareness, even though the bi-stable visual phenomenon is a classic experimental paradigm for investigating the NCCs $(6-8)$. There has been some suggestion in the literature that the DMN may play a role in BR. In many fMRI/EEG studies utilising the BR paradigm, frontal and parietal regions have been reported to be 
engaged (28-31), some of which may overlap with DMN subregions, especially in the parietal cortex where high-level cognitive networks mostly converge (32). A hypothesis of the DMN's involvement in the BR task seems to be contradictory to its frequently attributed role of "task-negative", a characterisation that originated from its discovery where its activity at rest was found to be higher compared to a cognitively demanding task conditions (22). However, the perceptual experience during BR is no more cognitivelydemanding than the control condition which is usually a normal viewing condition. In addition, there has been substantial evidence showing DMN engagement in tasks, suggesting that contribution to cognitive function from areas that make up the DMN is still not well understood (3335). Therefore, here we aim to confirm the DMN's involvement during BR before answering how it exerts an influence; this constitutes the first part of the study. In fact, a recent fMRI study using ambiguous images as stimuli to study pattern disambiguation has established a role for the DMN in prior-guided visual perception (36), which suggests that the DMN is involved in the online modulation of visual processing and its function may perhaps be associated with perceptual disambiguation.

Given the DMN's role in global cortical dynamics and conscious representation, we hypothesised that the intrinsic DMN dynamics may have some influence on the involuntary perceptual fluctuations during BR, possibly by causing perturbation to the equilibrium of the current dominant percept.

\section{Results}

\section{Behavioural analyses}

For maximally eliciting BR perception, the experiment used stimuli of rotating green and red checkerboard images, which were presented to each eye simultaneously with the order counterbalanced across individuals. In a perceptually matched Replay (RPL) condition where no BR is elicited, the same rotating images of the red or green checkerboard were presented to both eyes at the same time, but they alternated in time (Fig. 1a, d). Participants were asked to instantaneously report their percept by using three different buttons, respectively for the (dominant) red or green percept, and the mixed percept i.e. a transitional phase between the red and green percept. Therefore, this paradigm had a $2 \times 2$ factorial design of dominant and mixed perception types for the BR and RPL conditions. 
Individual difference of the percept duration during BR was significant (Fig. 1b) (reduced$\mathrm{RSS}=1490, \mathrm{p}=0.00$ by a $\chi^{2}$ test; we also adopted the Akaike Information Criterion/AIC for statistical inference, which was decreased by 1057.5, suggesting stronger model evidence for a model considering individual differences compared to the one without it). The duration of (dominant/mixed) percept formedaheavy-tailed distribution (skewness $=1.29$, kurtosis $=2.89$ for dominant percept; 3.15 and 17.82 for mixed percept), and the dominant percept (median=2.22 seconds for the red and 2.04 for the green) was significantly longer (decreased AIC/dAIC $=7985)$ than the mixed percept (median=0.33) (Fig. 1c). Although stimulus colour seemed to be able to slightly influence perceptual duration according to the statistics, we do not explore this effect further and refer to the stable perception for both colours as the dominant percept. In fact, a subsequent fMRI contrast did not reveal significant activation difference between red and greenpercept. 
a
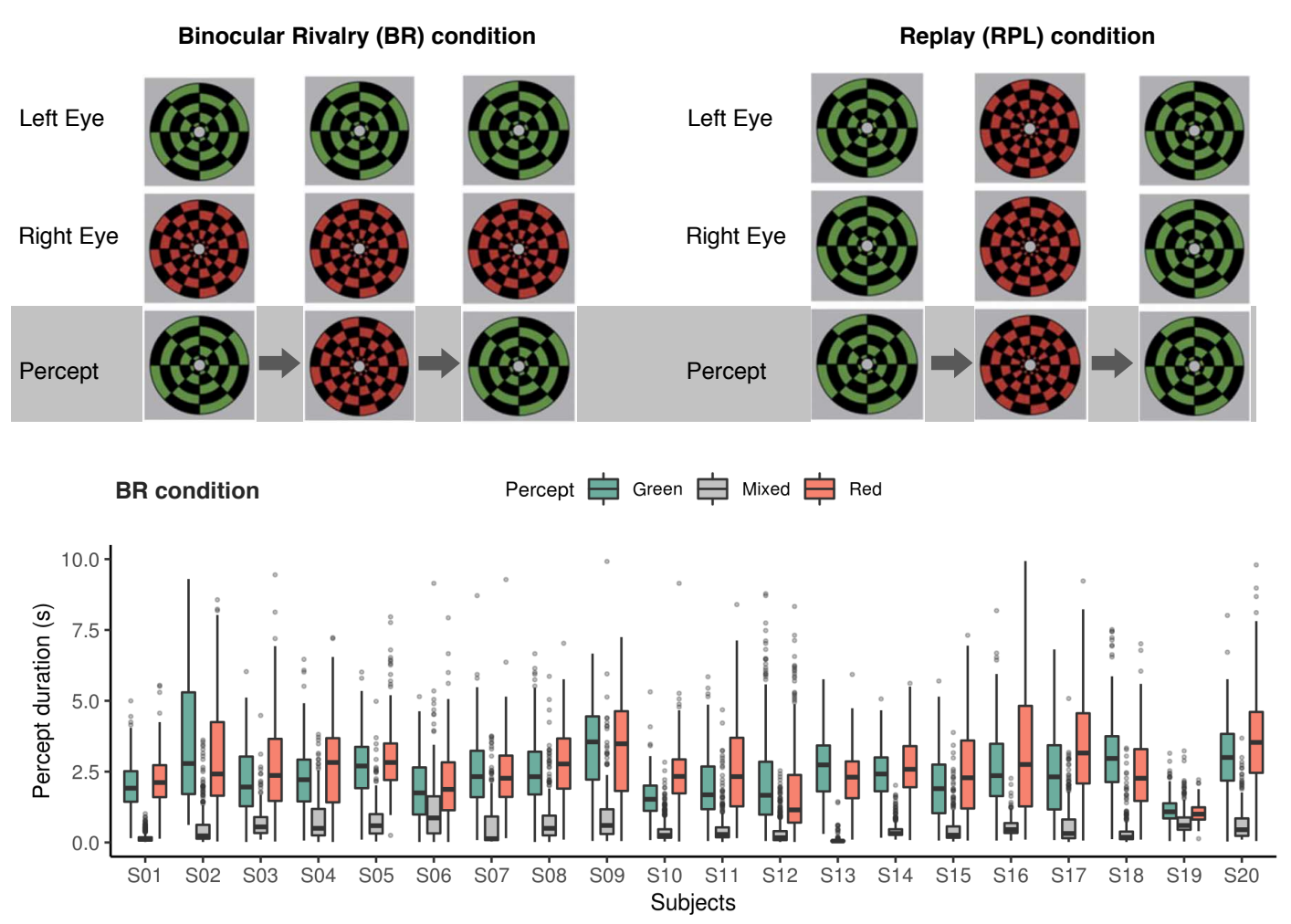

C

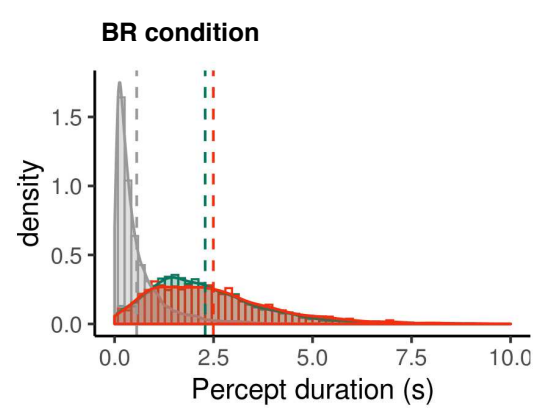

d
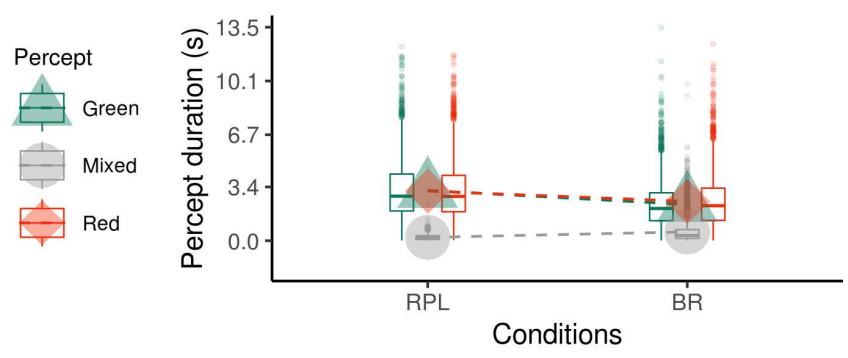

Fig. 1. Experimental design with 2-by-2 conditions of different percepts and ways of percept generation (a) Experimental schema adapted from the original study (37) from which the data was shared. During the binocular rivalry (BR) blocks (left), the participants were presented with different images, each for one eye, in the centre of the visual field. During the replay (RPL) blocks (right), the participants were presented with the same image for both eyes, but this image alternates over time between red, green via a short superimposition, in order to simulate the experience in the BR condition. Both conditions can generate alternating percept of the dominant (red or green) and the transitional mixed images, however the perceptual alternation was generated endogenously in the BR condition while it was exogenously elicited in the RPL condition. (b) Individual difference in percept duration (s) for different percept types in the BR condition. 
(c) Distributions of percept duration in the BR condition. (d) Percept duration for different percepts in the RPL and BR condition.

\section{FMRI activation study}

In agreement with the previous literature $(30,31)$, we found significantly activated regions in the cuneus, the intraparietal sulcus/inferior parietal lobule (BA40), angular gyrus (BA39) and inferior frontal gyrus (BA47), for the contrast of BR (dominant) > RPL (dominant). We also replicated the finding of the deactivation in the posterior (mid-) cingulate cortex (BA31) upon a report of mixed percept during BR, which was referred to as "perceptual transition" in the original paper where the data was shared from (37). As suggested by the meta-analysis driven BrainMap network atlases, a large portion of the significantly activated regions over- lapped with the DMN, supporting our hypothesis about the DMN's involvement in the task (see a full report of significant clusters in the Supplementary Materials). Here we highlight an F- contrast for the 2-by-2 interaction effect: 2 perception types (dominant vs. mixed) $\times 2$ ways of perception generation (BR vs. RPL), which revealed the DMN and visual cortex having the most variable activation patterns among the four conditions (Fig. 2). Our subsequent dynamic analysis will mostly focus on these regions. 

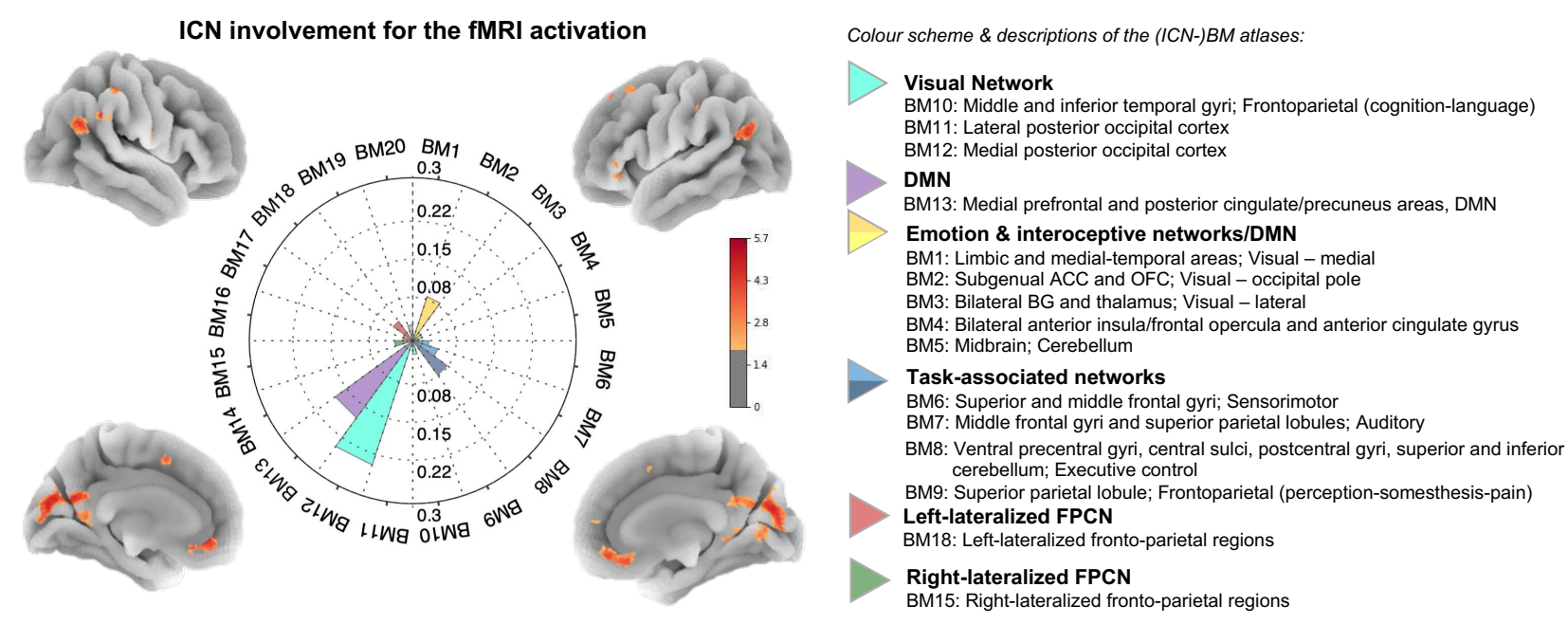

Fig. 2. ICN affiliation of the fMRI activated regions as revealed by the interaction effect. The interaction analysis reveals the regions whose activity is most sensitive to the four conditions, i.e. the regions mostly engaged by the experiment paradigm. The brain activation maps indicate the significant regions that showed an interaction effect among the 4 conditions, passing the cluster-level threshold of 0.05 (family-wise error corrected/FWE-corr) upon an F test (two-tailed). ICN involvement is a measure of correspondence between an activation map and large-scale networks with well-established cognitive function. The spatial correspondence is supported by the BrainMap meta-analysis database (38).

\section{EEG evoked responses and source reconstruction}

The EEG recording with its excellent temporal resolution gave us the opportunity to better locate the neuronal cause of a perceptual change by looking into a short time interval right before the perceptual change. Permutation cluster tests on the Global Field Power (GFP) of the perceptual conditions revealed that the most eventful epoch was between [-400-200] ms, which was when the EEG sensor voltages differed the most between the endogenously (BR) and exogenously (RPL) generated dominant percept (permutation cluster test on the time-window $[-500,0] \mathrm{ms}, \mathrm{p}<0.05$ ) (Fig. 3a). We observed no significant difference for mixed percept between the BR and RPL conditions during the same temporal window (Fig. 3b). Further 2-way ANOVA analysis in this time-window revealed a significant main effect of the source of perceptual transitions $(\mathrm{F}(1,19)=4.70, \mathrm{p}=0.04)$, but no significant main effect of perceptual types 
(mixed or dominant) was found $(F(1,19)=0.29 ; \mathrm{p}=0.59)$. Although there was a noticeable trend of interaction effect between the source of perceptual transitions and the perceptual type, the test was not significant $(F(1,19)=2.63 ; p=0.12)$ (Fig. 3c). The event-related potential (ERP) topographies during the $[-400-200]$ ms time window for the dominant percept in both conditions are shown in the Fig. $3 \mathrm{~d}$.

To discover the brain location of the signals that generated this scalp topological difference, we then conducted source reconstruction for the ERPs during this time window. The source reconstruction indicated that the topology was driven by a deactivation in the posterior cingulate cortex (PCC; BA23/24), precuneus (PCU; BA31/7m), thalamus, insula, caudate, claustrum and the fusiform gyrus (BA20) during BR (dominant) vs. RPL (dominant) condition (full results presented in the Supplementary Materials). An F contrast during this time window for the interaction effect of the 4 conditions revealed the following regions: insula (BA 13), postcentral gyrus (BA 43), thalamus, parahippocampal gyrus (BA 34), PCC (BA30), inferior frontal gyrus (BA 47) and anterior cingulate cortex (ACC) (Fig. 3e). See supplementary materials for a full report of significant regions, including relevant statistics and the results for other contrasts, which were classified into finer grained frequency bands and time windows. 

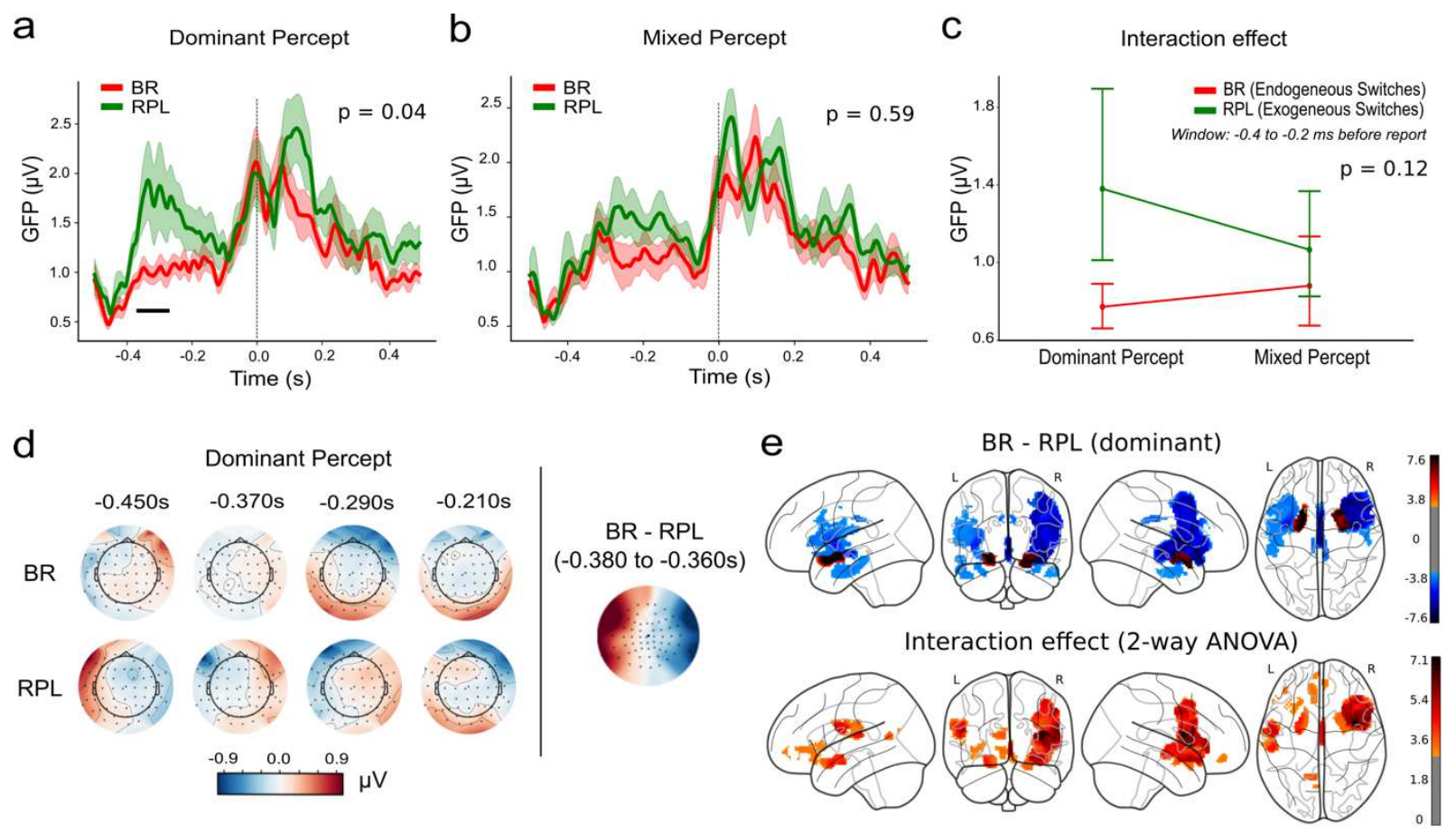

Fig. 3. Event-Related Potentials for different perceptual conditions. (a) Event-Related Potentials for different perceptual conditions at the sensor level. The global field power (GFP) in the replay condition was significantly higher than that in BR condition from 400-200 ms before the report (at the $0 \mathrm{~s}$ ) of change in percept (black line: $p<0.05$ significant clusters, 1-sample permutation cluster t-test). (b) No significant difference in the overall GFP during mixed percept between the BR and RPL conditions. (c) GFP difference across sources of perceptual transitions and types of percept. There was a significant main effect of the source of perceptual transitions (BR/RPL) (2-way ANOVA, p <0.05). (d) ERP topographies for dominant percept in both of the BR and RPL conditions, and the contrast between them. (e) The localisation of (relative) evoked responses at the source level, contrasted between BR (dominant) and RPL (dominant) conditions, and for the interaction effect of the two variables.

The EEG evoked response and the BOLD response for the interaction effect with the experimental conditions largely overlap in the ACC, PCC, inferior frontal gyrus and the precentral gyrus. However, cuneus (BA18), extrastriate cortex (BA19) and posterior parietal cortices (PPC; BA39/40) only showed an interaction effect in the BOLD response but not in the EEG evoked response. This might be due to the fact that the EEG evoked response captured the moment before the participant's explicit response for a perceptual change, while the fMRI activation should be mainly driven by the effect after the perceptual change. During the pre-response window for an immediate perceptual change, the visual 
percept might not have actually changed, so no significant cross-condition difference in the EEG evoked response was found in the visual areas, nor should there be a change of attention level (as indicated by the PPC BOLD activity) yet in this time. But an elevated level of attention could be caused by a change of perception by the time the participants already reported a perceptual change, and this might be what the fMRI activation in the occipital and posterior parietal cortices represented.

\section{Hidden neural states within DMN can predict upcoming perceptual transitions}

The previous evoked-response analysis showed that the biggest difference between BR and RPL conditions happened right before ( $\sim 1$ second) perceptual transitions. However, the evoked response of the GFP is a crude measure as it is a grand average across all trials and all channels. To uncover the dynamic patterns embedded in the EEG signals on a trial-by-trial basis, we adopted the embedded-lag HMM method (https://github.com/OHBA-analysis/HMM-MAR) which takes advantage of the excellent temporal resolution and rich spectral profiles of the EEG data. If the intrinsic fluctuation of the DMN regional activity influences the perceptual transitions during BR, we should expect to find a correspondence between the temporal dynamics of DMN regions and the upcoming perceptual transitions. To increase the sensitivity of the method as well as to avoid overfitting, we constrained our regions of interest to those which were shown to be significantly engaged in the experiment by the source-level evoked EEG activation contrasts between experimental conditions. These regions were DMN key regions, including bilateral parahippocampal gyri (HP), bilateral inferior parietal lobules (IPL), ACC, PCC, PCU, and the primary visual cortex (V1) (a full list of the regional coordinates where the signals were extracted is presented in the Supplementary Table 1). We targeted our dynamic analyses at the temporal window of the second (with 0.1 -s post-response padding) before every perceptual transition as a trade-off between having enough sample points and having enough specificity for the targeted events.

We set the HMM algorithm to extract 4 states from the data, which are the concatenated regional signals of all trials from all conditions and subjects (Fig. 4). We chose 4 states because firstly we were interested in the differences of the neural activity among the four experimental conditions, supposing that the differences can be captured by the most salient four components in the variance of the data; 
and secondly, 4 components have often been chosen for discovering the EEG microstates in existing literature (39), and were shown to have correspondence with the ICN dynamics measured from the BOLD signals (40). These data-driven states were then examined and compared between the four experiment conditions. The resulting auto-covariance patterns of the 4 states are presented in the supplementary materials (sFig. 5). All of the comparisons were carried out within subjects and the difference was then grouped together for the population-level inference. 


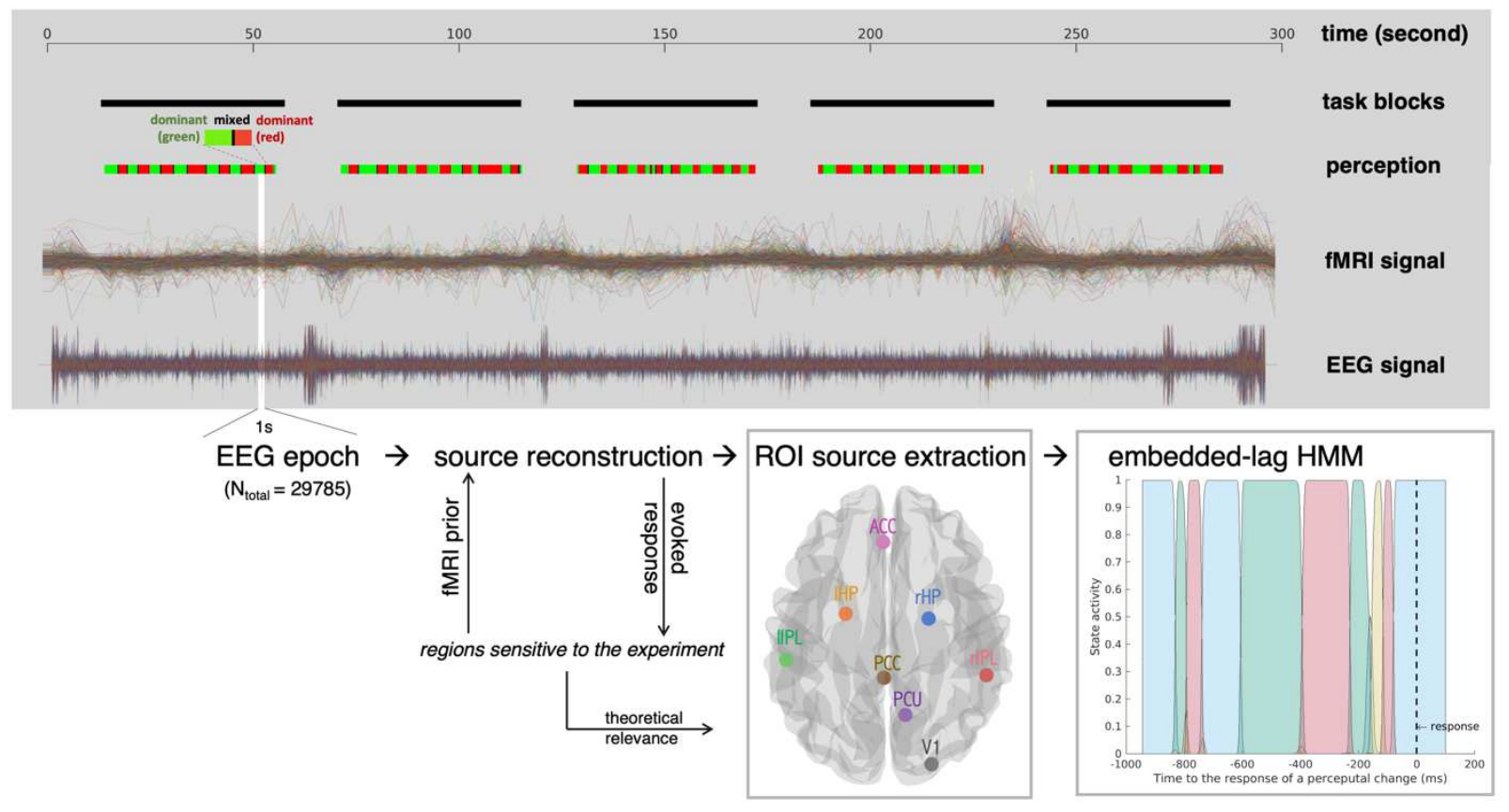

Fig. 4. Schematic illustration of the procedures for HMM. The one-second EEG epochs/trials were taken right before every perceptual change ("dominant" in red or green, "mixed" in black). To ensure experimental sensitivity, we select our regions of interest (ROI) from the significant results of the sensor-level evoked response in our previous analyses. Also because of our theoretical interest, we further constrained our selection of ROIs by choosing only the V1 and DMN regions to construct the HMM. Across all the experimental conditions, signals from significant peak voxels within the same ROIs were averaged for being used as the regional signal. The anatomical labels for the peak coordinates were identified with the Talairach Atlas (http://www.talairach.org/daemon.html), upon a conversion to the Talairach space. Full lists of the peak voxel coordinates used for this purpose are presented in the supplementary material.

We firstly compared the states' dynamics features, such as the switching rate (SR), i.e. the rate of state switches which can be understood as a measure of state stability, and the fractional occupancy (FO), i.e. the proportion of dwelling time on a certain state given a period of time. The SRs were not different across conditions, except that the RPL (mixed) had significantly higher SR than the rest ( $\mathrm{t}=3.57, \mathrm{p}$-adj $=0.00$ vs. BR (dominant); $\mathrm{t}=3.30, \mathrm{p}$ - $a d j=0.00$ vs. $\mathrm{BR}$ (mixed), and $\mathrm{t}=3.00, \mathrm{p}$ - $a d j=0.01$ vs. RPL (dominant)). The fact that the SRs were about a quarter each suggested that there was a good mix of the presence of the 4 states, meaning the 4 states had been separated well for capturing the multi-dimensional variance in the data.

Importantly, we found that the four states' FO was significantly different among the conditions $(\mathrm{p}=0.00, \mathrm{dAIC}=147.45)$. To establish exactly how the states' FO differed (Fig. 5a), permutation tests 
were conducted within each experimental condition for state-wise comparisons (with 2000 permutations for simulating a null distribution of the state-wise differences). It turned out that the states can discriminate conditions, in a way that a unique state was always visited mostly during one specific condition (Fig. 4b). This pattern was most noticeable in the BR (dominant) and RPL (mixed) conditions, where respectively State 4 and State 1 clearly stood out, with significant median differences of $0.44(\mathrm{p}-a d j=0.00)$, and $0.36(\mathrm{p}-a d j=0.00)$ to the second most prominent state in their respective conditions. Similarly, in the RPL (dominant) and the BR (mixed) condition, State 2 and State 3 were respectively visited the most, nevertheless they were not visited significantly more often than the second most prominent state.

We reflected on the reason why the RPL (dominant) and BR (mixed) conditions did not generate a single state as significantly dominant as was the case in the other two conditions. This might be explained by the idiosyncrasies of the perceptual compositions in some of the trials, given the nature of the experiment. In this experiment, the mixed percept can be seen as an ending phase of the last dominant percept, and a starting phase of the next one, and it is much shorter $(\sim 0.5 \mathrm{~s}$ on average $)$ than its adjacent dominant percept $(\sim 2 \mathrm{~s})$ (Fig. 1c,d). For data analyses, we extracted uniformly 1-s time windows before any perceptual switches to ensure equal (in terms of duration) data-points for the analysis. Therefore, the 1-s window before a dominant percept may cover periods of perceptual fluctuation, including the transitional mixed percept that is meant to be captured, and the residual of dominant percept from the last trial. The potential perceptual fluctuation in these trials could have confounded the RPL (dominant) condition, but it may not be a problem for the BR (dominant) condition. Because in RPL (dominant) condition, a perceptual switch was controlled by an external cause, as the images were designed to alter stochastically by the experimenters. By comparison, perceptual alternations in the BR conditions were generated endogenously, so the transient perceptual instability before may be considered as leading to a next dominant transition. Therefore, despite the BR (dominant) trials could contain perceptual fluctuation, the fluctuating percepts may be driven by same neural causal power and thus can be treated as common factors.

To validate the point that the continuity of the causal power in the neural activity is what sets the BR condition apart from the RPL condition, we further conducted a cross validation (CV), using the trial-by-trial FOs of the hidden neural states to predict the type (mixed or dominant) of the upcoming transition. This was done respectively for the BR (endogenous) and RPL (exogenous) 
conditions. As a result, the accuracy score for the BR condition was 92.01\% ( $\mathrm{SD}=4.68 \%)$, which was higher than the score of $82.16 \%(\mathrm{SD}=9.14 \%)$ for the RPL condition (see the Supplementary Material sFig. 15 for more details). This suggests that these neural states we found in a purely data-driven way, actually contain the causal power for predicting the endogenously driven perceptual changes. In addition, according to the state FOs for each of the conditions, there was a mirrored difference between the BR and RPL conditions. Namely, State 4 was visited distinctively the most in the BR (dominant) condition, but it was visited the least in the RPL (dominant) condition. Therefore, State 4 seems to be key to the intrinsic neural cause of the spontaneous perceptual fluctuation during BR. 


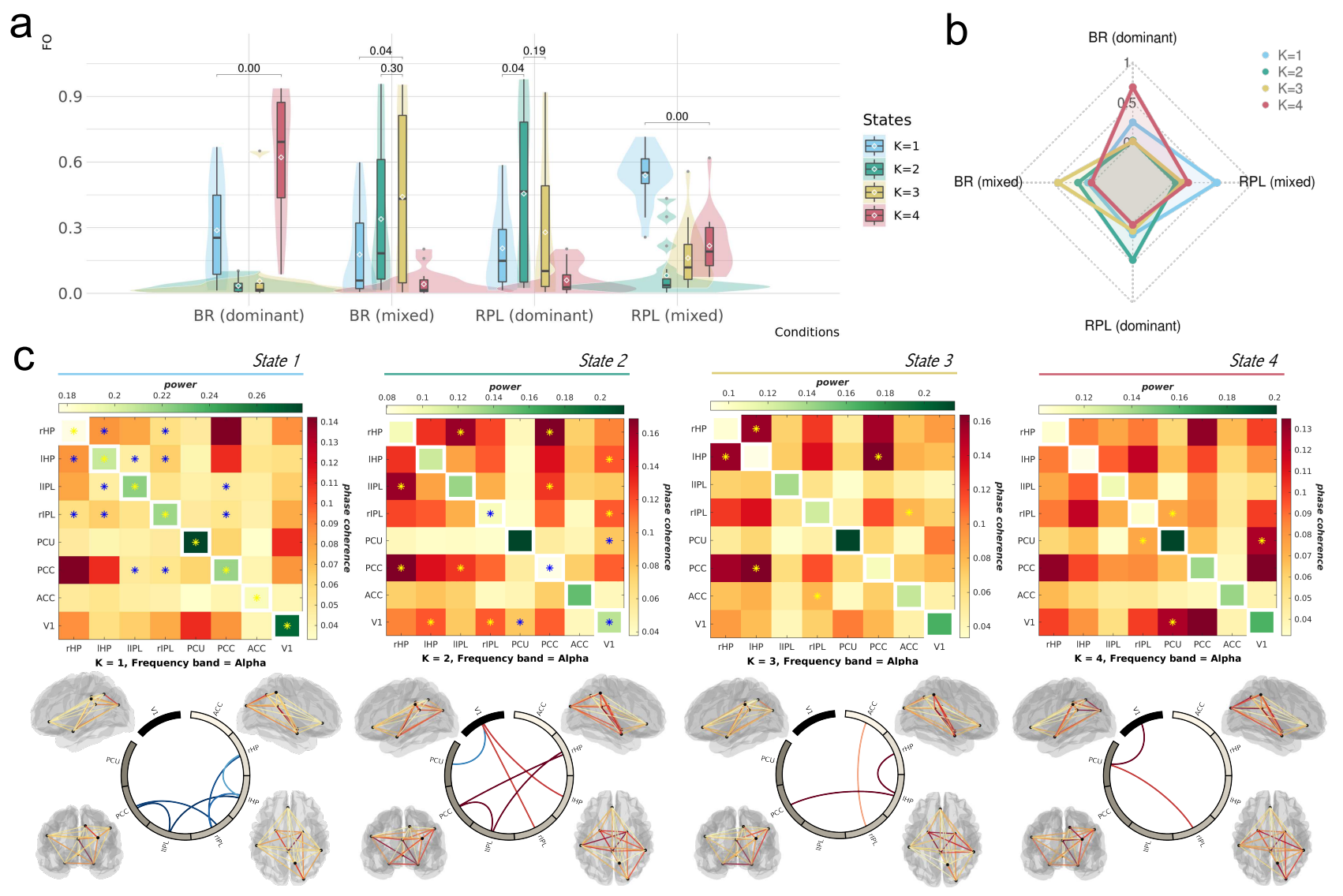

Fig. 5. Correspondence between states and conditions (a) Different fractional occupancy (FO) of the four states across conditions. Plotted data are the mean FO values averaged across trials for each individual, grouped by the two categorical variables: conditions and states. FDR-adjusted p-values of pair-wise permutation tests are presented on the significance bar on top of the compared groups. The distributions of non-averaged FO for all trials/epochs of all participants are presented in supplementary materials (sFig. 8). For the box plots, the white mark on each box indicates the mean, while the length of the box indicates quantiles with the middle bar showing the median. (b) Radar chart showing the correspondence between conditions and states. Scales on the radar chart indicate the median values of the state FO in the corresponding condition. (c) Spectral information of the states in the Alpha band. Diagonal and off-diagonal values of the upper-triangle heatmaps respectively indicate the power and phase coherence of the ROIs. The asterisk on the heatmap indicates that the phase coherence (or power) in this state is significantly higher (yellow) or lower (blue) compared with the other states (one-tailed test with the $95 \%$ confidence interval by permutation). The circular bundle plots below highlight the significant connectivities, with red and blue respectively signifying higher and lower significance for the connectivity. All of the connectivities (whether significant or not) are also mapped onto standardised brain anatomy from the left, right, back and top views (clock-wise), where the colour intensity of the connectivity has been normalised across all states, thus being suitable for visual state-wise comparisons. Coordinates of the nodes were selected based on significant peaks from the previous evoked-response analyses (See the Supplementary Table 1 for all of the peak coordinates). 


\section{PCU-V1 phase coherence and Granger Causality predicts stable percepts}

To unravel the neuronal signatures encapsulated in the states, we then extracted the spectral information (power and phase coherence) from the multivariate auto-covariance matrix of each state. Once we had estimated the power and coherence for each state, we factorised the frequencies into a few dominating components so as to facilitate interpretation with conventionally defined frequency bands. For that, we applied a non-negative matrix factorisation (NNMF) algorithm on the coherence matrix, concatenated across all states and ROIs and confined it to four components. The four components turned out to match traditional frequency bands: Theta (peak at $4 \mathrm{~Hz}$, half- maximum at 1 , $12 \mathrm{~Hz}$ ), Alpha (peak at $11 \mathrm{~Hz}$, half-maximum at 7, $15 \mathrm{~Hz}$ ), Beta (peak at $20 \mathrm{~Hz}$, half-maximum at 15, $34 \mathrm{~Hz}$ ) and Gamma (peak at $34 \mathrm{~Hz}$, half-maximum at $26 \mathrm{~Hz}$ ) (sFig. 7 in supplementary materials). Therefore, we next focused into state-wise frequency- specific spectral information. As stated already, the data-driven states were indicative of the 4 experimental conditions (representing 4 kinds of perceptual experiences). We then looked into how each state is represented by the spectral features of the neural signals. To identify the characteristics of each state that stand out, we performed permutation tests for each power and coherence values, comparing across all 4 states (5000 permutations for each null distribution simulation; significance level is $\mathrm{p}<0.01$ ) (Fig. 5c).

For a clear narrative, here we only focus on the Alpha band (peak at $11 \mathrm{~Hz}, \mathrm{FWHM}=8 \mathrm{~Hz}$ ) of the states. Previous studies have shown that pre-stimuli alpha oscillations at posterior sensors can modulate subsequent processing and perception (41). In our study, the alpha band of the states also demonstrated an interesting pattern in the state-wise comparisons. Comparing the phase coherence among the ROIs across the four states (Fig. 5c), the one between PCU and V1 clearly stood out, as the PCU-V1 phase coherence was highest in State 4, an indicative state for the BR (dominant); and it was the lowest in State 2, an indicative state for the RPL (dominant) condition. This made us hypothesise that the PCU-V1 coherence may underlie the spontaneous transition to a stable percept.

We have presented the full results of other frequencies in the supplementary materials (sFig. 1012). For the other frequencies, we would like to only point out the general increase of the theta coherences among DMN regions in the BR (mixed) condition (i.e. during a dominant percept because the trial is extracted before a perceptual change), especially the increased theta coherence between 
ACC and PCC, which is unseen in other frequencies. This corresponds to previous MEG literature where intrahemispheric and interhemispheric theta coherences were found to be increased during periods of perceptual dominance in BR (42). The strong ACC-PCC coherence that we only observed in the theta oscillations also corresponds to an earlier study using the same HMM method which showed that the mPFC/ACC-PCC coherence in the delta/theta model characterises the anterior higherorder cognitive state during resting state (19).

\section{PCU-V1 phase coherence and Granger Causality predicts stable percepts}

We then investigated the behavioural relevance of the critical state we found: State 4. State lifetime (also known as dwell-time) was used as an intuitive index for the state presence, estimated as the time that elapsed between entering and exiting a state according to the HMM (43). We correlated it with the reaction time (RT) which was defined as the interval of the participant's adjacent reports to perceptual changes. When the correlation is examined on the individual level (i.e., when the variance is provided by different performances across trials), the RT was the perceptual duration of the last percept; but when examined on the group level (i.e., variance provided by different average performances of the individuals), the individual- averaged RT reflected the alternation rate for that participant. We found that longer State 4's lifetime could predict faster perceptual transitions $(\mathrm{b}=-0.38, \mathrm{t}=-2.27, \mathrm{p}=0.03$; $\mathrm{dAIC}=35.03$ ) at the group level.

To better understand the cognitive implication of the PCU-V1 coupling, which was a main feature of State 4, we further investigated the causal effect of the PCU to V1 and its relationship with behavioural measures. As we were agnostic to the nature of this hypothetical causal relationship, we adopted a simple form of causality to perform this analysis, namely, Granger Causality, which basically searches for a time-lagged linear relationship between $\mathrm{x}_{1}$ and $\mathrm{x}_{2}$ that can predict $\mathrm{x}_{1}$ better than what $\mathrm{x}_{1}$ 's own auto-regression can predict (i.e., $\mathrm{x}_{2}$ granger-causes $\mathrm{x}_{1}$ ). In the $\mathrm{BR}$ literature, it is commonly believed that a switch of perception is caused by a slip of equilibrium due to neuronal noise, and/or the constant decay of suppression that one neural population has over the other, due to habituation effects $(10,11,44)$. Apart from these, attention effects have also been shown to play a role $(10,45)$. Therefore, the PCU $\rightarrow \mathrm{V} 1$ top-down modulation could reflect either an intrinsically evoked attention effect, or an effect of the global adaptation/perceptual habituation. These two possibilities would lead 
to opposite trial-by-trail predictions. The attention effect has been shown to speed up BR transitions, possibly by increasing the visual contrast of the percept (46). If the PCU $\rightarrow \mathrm{V} 1$ modulation represents the attention effect, we can predict that the stronger the causal effect is, the quicker the transition becomes. Whereas, if the PCU $\rightarrow \mathrm{V} 1$ modulation reflects the adaptation/habituation effect, longer duration of the last stable percept should cause a stronger adaptation (i.e. stronger $\mathrm{PCU} \rightarrow \mathrm{V} 1$ modulation) in the following trial.

We constructed statistical models according to the above hypotheses, but we did not find a trialby-trial relationship between the $\mathrm{GC}(\mathrm{PCU} \rightarrow \mathrm{V} 1)$ and the perceptual duration. However, the two variables were correlated on the group level. Specifically, we found an interaction effect between the individual-averaged state lifetime $(\mathrm{K}=4)$ and $\mathrm{GC}(\mathrm{PCU} \rightarrow \mathrm{V} 1)$ for predicting the individual-averaged perceptual duration. In other words, the GC becomes more relevant to quicker perceptual transitions when the trial is visited by State 4 more $(b=-0.39, t=-3.01, p=0.00$ fitted with a generalised linear model; $\mathrm{dAIC}=4.54$ compared to a multiple regression model with no interaction). When State 4 dwells for the majority $(>95 \%)$ of the trial time(i.e., $>0.95 \mathrm{~s})$, the negative correlation between the GC and the perceptual duration was significant $(b=-0.931, t=-2.821, p=0.017$; $d A I C=29.56)$ (All statistical models have been presented in the Supplemental Materials).

The $\mathrm{GC}(\mathrm{PCU} \rightarrow \mathrm{V} 1)$ may be interpreted as an averaged modulation effect of the PCU to V1during the 1-second window of concern. That only its interaction with the lifetime of State 4 is significant for predicting perceptual fluctuations suggests that: 1. The modulation effect of the PCU to $\mathrm{V} 1$ is perceptually relevant, but it alone is not sufficient for leading to a perceptual transition. A perceptual transition also depends on the presence of State 4, which consists of many other features over and above the PCU-V1 relationship (e.g. the phase coherence between PCC and V1 in the theta and beta band) which were not discussed in this paper and were presented in the supplementary materials sFig. 10-12); 2. The modulation effect of the PCU to V1 seems to be temporally accumulative along with the lifetime of State 4, suggesting that it needs to be built up and pass a certain threshold to take effect. 
a
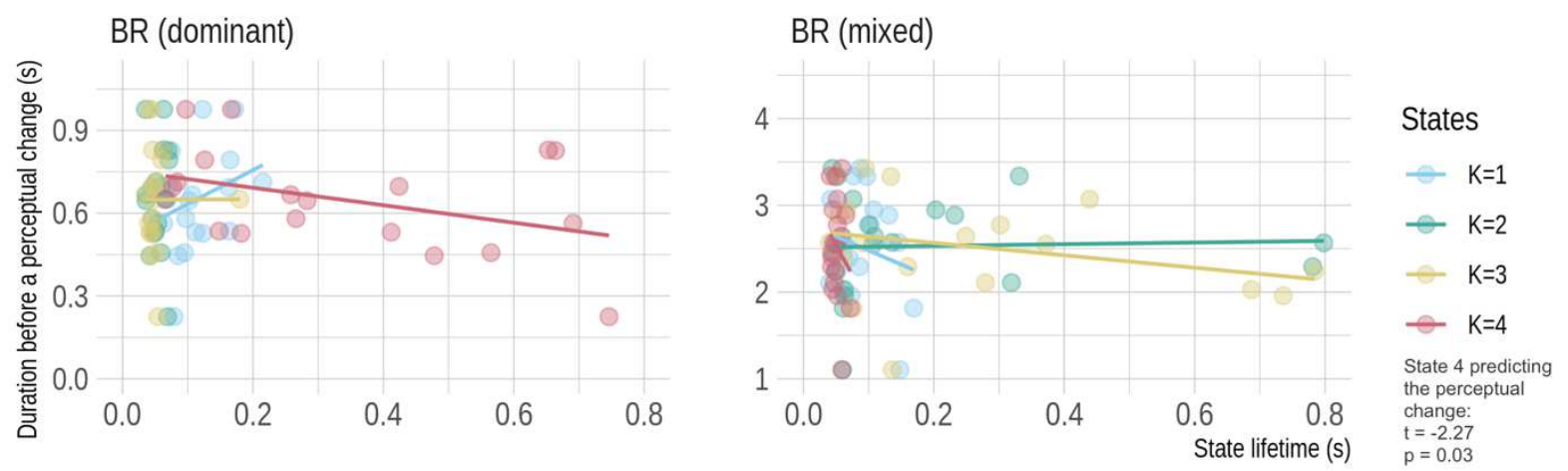

b
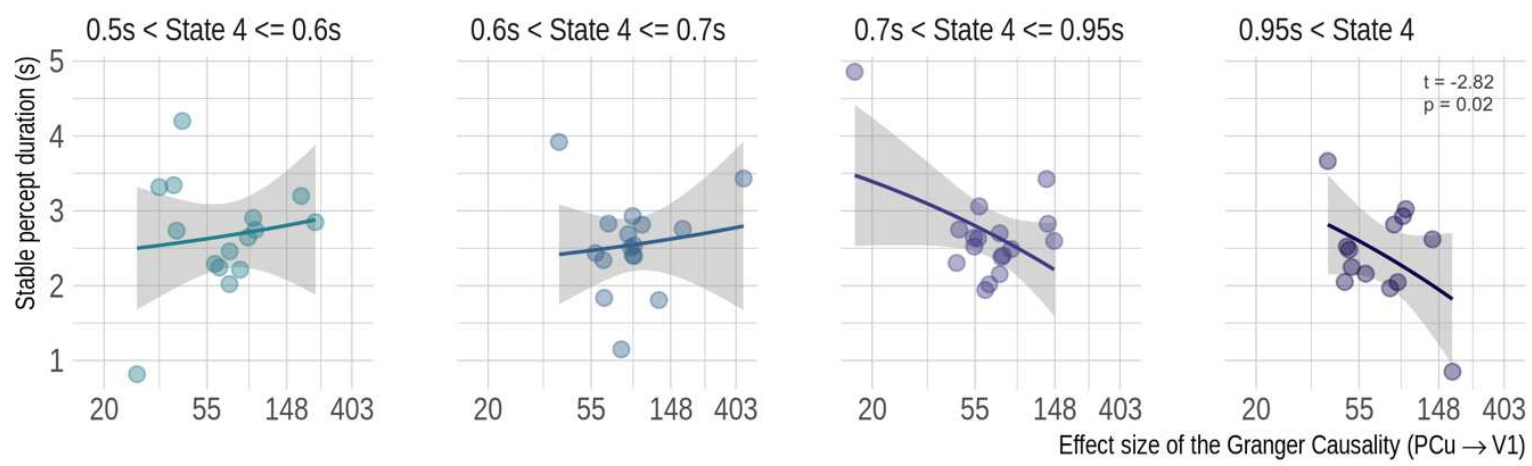

Fig. 6. (a) Lifetime of State 4 can predict the length of intervals between perceptual changes during BR (for both dominant and mixed transitions). The longer State 4 persists, the shorter the transitional period lasts, i.e. the quicker the rivalry is resolved. (b) shows that the duration of the last stable percept is correlated with the Granger causality (GC) effect from PCU to V1 on the group level. Their relationship is also dependent on the lifetime of State 4. The GC effect size was approximated by the improvement of model evidence $(\mathrm{dBIC})$. 


\section{Discussion}

Awareness is suggested to emerge through the interactions between local processing triggered by external stimuli, and global broadcasting afforded by the brain's intrinsic spatiotemporal states (1). Resting-state whole-brain signals are shown to be spatiotemporally organised (16), but it is still not clear whether this intrinsic architecture is epiphenomenal or functional. By using simultaneously recorded EEG-fMRI data, we showed that the brain's spatiotemporal patterns or states found in this study, characterised by EEG oscillation patterns in the DMN regions, are phenomenologically relevant. We found that the four data-driven states are associated with the four perceptual experiences corresponding to the experimental conditions. The state indicating a dominant perceptual transition during the BR task was characterised by phase coupling between the precuneus (PCU) and the primary visual cortex (V1). We further found that the causal effect from the PCU to the V1 is a temporally cumulative effect along with the state lifetime of State 4; namely, the interaction of them is associated with the rate of perceptual transitions. Taken together, our results suggest that the brain's intrinsic dynamics can influence visual awareness, possibly by triggering a diversion of the whole visual processing pathway.

There are a lot of controversies in existing literature regarding the sources of perceptual fluctuations during BR. Many advocate that the rivalry is resolved at the early visual stage, for example in the lateral geniculate nucleus or the primary visual cortex, probably due to sensory bottlenecks (4749). But the contradictory argument is also convincing. Tong et al. (1998) using "face vs. house" binocular stimuli suggested that the rivalry continues from the retina, V1, V4 and MT, until it reaches the object-based processing step, i.e., the human fusiform face area and parahippocampal place area as exemplified in their study (50). It is also a well-known phenomenon that the BR alternation rate can be modulated by object features, emotional or semantic loading embedded in the stimuli as well as higher-level cognition such as working memory and attentional control (51). Therefore, many supported a top-down control hypothesis, which suggests that the observed activity in the early visual pathway is but an outcome of the feedback projections from higher-level areas, while those higherlevel areas are where the NCCs actually lie $(52,53)$. According to them, the higher-level regions suppress unfavourable stimuli representation in the early visual pathway in order to maintain a congruent conscious experience (14). These high-level regions have been localised to frontoparietal 
regions by previous functional and structural MRI studies, including the frontal eye field, superior/middle/inferior frontal gyrus and superior/inferior parietal lobule (SPL/IPL) (54-56). Especially the right-lateralised SPL was suggested to play a causal role in the BR transition by a TMS study (54).

Despite its plausibility, the top-down control hypothesis cannot explain the BR phenomenon fully. Firstly, it is difficult to dissociate the cause and effect of the BR perception in practice. Given the poor temporal resolution of fMRI, the frontoparietal regions revealed by fMRIactivation studies might reflect an elevated level of attention drawn by a perceptual change during BR. The right lateralisation of the SPL, as often reported in the BR literature, suggests that it may get involved by association with attention (56). In a more recent BR study, when more accurate temporal information of perceptual transitions was provided, the significance of SPL disappeared (37). This also suggested that the SPL activity may be secondary to BR transitions, and that it might get involved via an attention effect which inevitably intertwines with involuntary perceptual change. Similarly, it has been argued that the involvement of the frontal regions during BR may also reflect introspection and action to perceptual transitions, rather than being a real cause $(57,58)$. Even if the involvement of the frontoparietal regions represents the endogenous attention which is necessary for gating visual awareness, it has been shown that attention is a dissociable factor to visual awareness; therefore, it is still arguable whether the frontoparietal regions associated with an attentional effect can be considered as a true $\mathrm{NCC}(6,31,59)$. Secondly, the top-down control hypothesis cannot explain why the perceptual transition keeps occurring when a stable percept has been reached. According to this hypothesis, the rival signal is suppressed in the early visual pathway so that the higher-level regions can maintain a meaningful representation of the environment; then under the condition of a stable percept already being achieved, it would not make sense for the neural activity to suddenly revolt and take another route.

Our study provides a novel perspective as to why regular perceptual transitions may occur during BR. We highlight the possible influence of the temporal unfolding of the intrinsic large-scale cortical dynamics, which could bias the binocular signalling gains, perturb the current equilibrium, and eventually lead to a diversion of visual stream. Vidaurre et al. (2018) found that the intrinsic transient states exhibited in the posterior subdivisions of the DMN were characterised by high power and total coherence in the alpha $(8-12 \mathrm{~Hz})$ range (19). It was also suggested that pre-stimulus posterior 
alpha power can modulate primary visual processing, likely by re-shaping the functional architecture of the brain network when preparing for upcoming processing (41). These findings support our hypothesis that the DMN's activity may serve as a source of "perturbation" to a bi-stable visual system during $\mathrm{BR}$, thus introducing a bias towards what external information gets released from the overloaded visual channel. In our study, we revealed 4 states in the EEG signals from the DMN nodes and V1, during the one-second time window before a perceptual alternation. The 4 brain states, found in a datadriven way, turned out to be phenomenologically meaningful, for their differential presence during the "pre-onset" window could predict the different types of the upcoming perceptual transitions; and the lifetime of the critical state (State 4) correlates with the perceptual-alternation rate on the group level. The critical state we found also exhibits important features regarding the brain connectivity. One of the dominant features we followed up in this study is the phase coherence between PCU and V1 in alpha oscillations, as it seems to underlie the dominant perceptual transition in BR. Phase coherence measures the consistency of the phase differences between two waves across trials. If there are consistent phase differences between two sources across many trials, it means the two neuronal populations always discharge in a temporally coordinated manner, i.e. a signal coupling (60).

Although the DMN and V1 are situated at the two opposite ends of the brain's functional gradient (23), our result suggests that they may achieve signal coupling via the PCU. To further understand this signal coupling, we have also derived a Granger-causal effect of the PCU to V1 from their EEG signals at the same time windows. We found that, during the critical state, the stronger the top-down effect from the PCU to V1, the quicker the individual's perception alternated. However, this relationship only existed on the group level, but not for trial-by-trial prediction; therefore, it only establishes the behavioural relevance of the causal effect of PCU to V1, but did not provide enough evidence for us to claim that the causal effect from PCU to V1 means either signal adaptation or suppression, as both could potentially lead to a trial-by-trial prediction. That neither state properties nor Granger-causality from PCU to V1 could predict trial-by-trial performance may just be due to a lack of statistical power; however, it could also be a true negative. Namely, the intrinsic brain dynamics may reflect more of individual-specific biological regularities, rather than "on-line" cognitive control. In fact, the influence of voluntary top-down control over BR is known to be limited (45, 61, 62); and BR has been reported to have notable inter-individual differences, depending on the individual's gender, psychological predispositions, neurotransmitter levels and even handedness $(5,51)$. Twins and genomewide association studies have showed that the individual differences in BR can be attributed to genetic 
factors $(63,64)$. These suggest that BR may be bound to certain biological constraints of the brain's general state, while participants' involuntary top-down control over the BR rate as exemplified in previous studies (such as more intrinsic attention evoked by higher contrast of the image) might have exerted influence rather indirectly by altering the general state of the brain. Therefore, in spite of the PCU's well-known cognitive relevance to visual-spatial imagery (65), we suggest that its role in BR transition does not necessarily correspond to voluntary cognitive processes, either attentional control or memory retrieval $(62,66)$. Rather, the PCU-V1 coupling demonstrated in this study could be an indication of the intrinsic dynamics making "ripples" onto the primary visual cortex.

Finally, we would also like to comment on the correspondence between the brain's spatiotemporal dynamics and the phenomenological mental dynamics. With abundant empirical evidence and theoretical arguments, it has been suggested that the spatiotemporal patterns of neural dynamics are the instantiation of mental dynamics $(17,67)$. We also believe that the subjective experience is nothing more and nothing less than neural activities which are manifested at the mental level. But this does not necessitate the case that the emerged patterns in the physical domain (e.g. the spatiotemporal dynamics in neural signals) and those in our mental domain (e.g. human cognition) should have a linear mapping. Take our BR results for example, the transient states (short-lasting for $100 \mathrm{~ms}$ ) we found in the 1-second period before a subjective report were the spatiotemporal patterns corresponding to the upcoming perceptual changes, but participants were typically not aware of any change before its sudden occurrence. This further made us reflect on the function of the DMN. Evidence has suggested that DMN is important for self-representation and consciousness $(26,68,69)$, but its dynamical patterns of activity may not necessarily correspond to the person's stream of consciousness in a simultaneous and linear fashion. However, the simultaneous and linear correspondence is always assumed in neuroscience where one of our biggest endeavours is to map between the brain and the mind. As the controversy about the DMN's function is accumulating (33), we probably need to rethink the legitimacy of the correspondence we are tempted to make between the physical and mental levels of existence. 


\section{References}

1. G. A. Mashour, P. Roelfsema, J.-P. Changeux, S. Dehaene, Conscious Processing and the Global Neuronal Workspace Hypothesis. Neuron 105, 776-798 (2020).

2. G. Deco, J. Cruzat, J. Cabral, G. M. Knudsen, R. L. Carhart-Harris, P. C. Whybrow, N. K. Logothetis, M. L. Kringelbach, Whole-Brain Multimodal Neuroimaging Model Using Serotonin Receptor Maps Explains Non-linear Functional Effects of LSD. Current Biology 28, 3065-3074.e6 (2018).

3. S. M. Smith, P. T. Fox, K. L. Miller, D. C. Glahn, P. M. Fox, C. E. Mackay, N. Filippini, K. E. Watkins, R. Toro, A. R. Laird, C. F. Beckmann, Correspondence of the brain's functional architecture during activation and rest. Proc Natl Acad Sci U S A 106, 13040-13045 (2009).

4. S. Lehky, Binocular Rivalry is not Chaotic. Proceedings. Biological sciences / The Royal Society 259, 71-6 (1995).

5. J. W. Brascamp, M. W. Becker, D. Z. Hambrick, Revisiting individual differences in the time course of binocular rivalry. Journal of Vision 18, 3-3 (2018).

6. R. Blake, J. Brascamp, D. J. Heeger, Can binocular rivalry reveal neural correlates of consciousness?

Philos Trans R Soc Lond B Biol Sci 369 (2014).

7. F. Crick, C. Koch, A framework for consciousness. Nat Neurosci 6, 119-126 (2003).

8. F. Crick, C. Koch, Seminars in the Neurosciences (Saunders Scientific Publications, 1990), vol. 2 , pp. $263-275$.

9. J. Seely, C. C. Chow, Role of mutual inhibition in binocular rivalry. Journal of Neurophysiology 106, 2136-2150 (2011). 
10. D. Roumani, K. Moutoussis, Binocular rivalry alternations and their relation to visual adaptation. Front Hum Neurosci 6 (2012).

11. P. Theodoni, T. I. Panagiotaropoulos, V. Kapoor, N. K. Logothetis, G. Deco, Cortical Microcircuit Dynamics Mediating Binocular Rivalry: The Role of Adaptation in Inhibition.

Front. Hum. Neurosci. 5 (2011).

12. M. A.Webber, P. C. Bressloff, The effects of noise on binocular rivalry waves: A stochastic neural field model. J. Stat. Mech. 2013, P03001 (2013).

13. R. Moreno-Bote, J. Rinzel, N. Rubin, Noise-induced alternations in an attractor network model of perceptual bistability. J. Neurophysiol. 98, 1125-1139 (2007).

14. R. Zhang, S. A. Engel, K. Kay, Binocular Rivalry: A Window into Cortical Competition and Suppression. J Indian Inst Sci 97, 477-485 (2017).

15. J. Hohwy, A. Roepstorff, K. Friston, Predictive coding explains binocular rivalry: An epistemological

review. Cognition 108, 687-701 (2008).

16. D. Vidaurre, S. M. Smith, M. W. Woolrich, Brain network dynamics are hierarchically organized in time. PNAS 114, 12827-12832 (2017).

17. G. Northoff, S. Wainio-Theberge, K. Evers, Is temporo-spatial dynamics the "common currency" of brain and mind? In Quest of "Spatiotemporal Neuroscience". Physics of Life Reviews 33, 34-54 (2020).

18. D. Vidaurre, A. J. Quinn, A. P. Baker, D. Dupret, A. Tejero-Cantero, M. W. Woolrich, Spectrally resolved fast transient brain states in electrophysiological data. NeuroImage $126,81-95$ (2016).

19. D. Vidaurre, L. T. Hunt, A. J. Quinn, B. A. E. Hunt, M. J. Brookes, A. C. Nobre, M. W. 
Woolrich, Spontaneous cortical activity transiently organises into frequency specific phasecoupling networks. Nature Communications 9, 2987 (2018).

20. M. W. Cole, T. Ito, D. S. Bassett, D. H. Schultz, Activity flow over resting-state networks shapes cognitive task activations. Nat. Neurosci. 19, 1718-1726 (2016).

21. M. E. Raichle, The Brain's Default Mode Network. Annual Review of Neuroscience 38, 433-447 (2015).

22. M. E. Raichle, A. M. MacLeod, A. Z. Snyder,W. J. Powers, D. A. Gusnard, G. L. Shulman, A default mode of brain function. PNAS 98, 676-682 (2001).

23. D. S. Margulies, S. S. Ghosh, A. Goulas, M. Falkiewicz, J. M. Huntenburg, G. Langs, G. Bezgin, S. B. Eickhoff, F. X. Castellanos, M. Petrides, E. Jefferies, J. Smallwood, Situating the default-mode network along a principal gradient of macroscale cortical organization. PNAS 113, 12574-12579 (2016).

24. S. Gu, F. Pasqualetti, M. Cieslak, Q. K. Telesford, A. B. Yu, A. E. Kahn, J. D. Medaglia, J. M. Vettel, M. B. Miller, S. T. Grafton, D. S. Bassett, Controllability of structural brain networks. Nat Commun 6, 8414 (2015).

25. X. Liu, H. Li, F. Luo, L. Zhang, R. Han, B. Wang, Variation of the default mode network with altered alertness levels induced by propofol. Neuropsychiatr Dis Treat 11, 2573-2581 (2015).

26. C. D. Perri, M. A. Bahri, E. Amico, A. Thibaut, L. Heine, G. Antonopoulos, V. CharlandVerville, S. Wannez, F. Gomez, R. Hustinx, L. Tshibanda, A. Demertzi, A. Soddu, S. Laureys, Neural correlates of consciousness in patients who have emerged from a minimally conscious state: A cross-sectional multimodal imaging study. The Lancet Neurology 15, 830-842 (2016). 
27. A. Vanhaudenhuyse, Q. Noirhomme, L. J.-F. Tshibanda, M.-A. Bruno, P. Boveroux, C. Schnakers, A. Soddu, V. Perlbarg, D. Ledoux, J.-F. Brichant, G. Moonen, P. Maquet, M. D. Greicius, S. Laureys, M. Boly, Default network connectivity reflects the level of consciousness in non-communicative brain-damaged patients. Brain 133, 161-171 (2010).

28. E. D. Lumer, K. J. Friston, G. Rees, Neural Correlates of Perceptual Rivalry in the Human Brain. Science 280, 1930-1934 (1998).

29. P. Sterzer, A. Kleinschmidt, G. Rees, The neural bases of multistable perception. Trends in Cognitive Sciences 13, 310-318 (2009).

30. G. Rees, G. Kreiman, C. Koch, Neural correlates of consciousness in humans. Nat Rev Neurosci 3, 261-270 (2002).

31. T. W. Webb, K. M. Igelstr"om, A. Schurger, M. S. A. Graziano, Cortical networks involved in visual awareness independent of visual attention. Proc. Natl. Acad. Sci. U.S.A. 113, 13923-13928 (2016).

32. R. M. Braga, D. J. Sharp, C. Leeson, R. J. S. Wise, R. Leech, Echoes of the brain within default mode, association, and heteromodal cortices. J. Neurosci. 33, 14031-14039 (2013). 33. F. Callard, D. S. Margulies, What we talk about when we talk about the default mode network. Front Hum Neurosci 8 (2014).

34. D. Vatansever, D. K. Menon, A. E. Manktelow, B. J. Sahakian, E. A. Stamatakis, Default Mode Dynamics for Global Functional Integration. J. Neurosci. 35, 15254-15262 (2015).

35. R. N. Spreng, The Fallacy of a "Task-Negative" Network. Front Psychol 3 (2012).

36. C. Gonz'alez-Garc'ia, M. W. Flounders, R. Chang, A. T. Baria, B. J. He, Content-specific activity in frontoparietal and default-mode networks during prior-guided visual perception. eLife 7, e36068 (2018). 
37. A. V. Roy, K. W. Jamison, S. He, S. A. Engel, B. He, Deactivation in the posterior midcingulate cortex reflects perceptual transitions during binocular rivalry: Evidence from simultaneous EEG-fMRI. NeuroImage 152, 1-11 (2017).

38. L. R. Koza'k, L. A. van Graan, U. J. Chaudhary, A' . G. Szabo', L. Lemieux, ICN Atlas: Automated description and quantification of functional MRI activation patterns in the framework of intrinsic connectivity networks. Neuroimage 163, 319-341 (2017).

39. D. Lehmann, R. D. Pascual-Marqui, C. Michel, EEG microstates. Scholarpedia 4, 7632 (2009).

40. J. Britz, D. Van De Ville, C. M. Michel, BOLD correlates of EEG topography reveal rapid resting-state network dynamics. NeuroImage 52, 1162-1170 (2010).

41. O. Jensen, A. Mazaheri, Shaping Functional Architecture by Oscillatory Alpha Activity: Gating by Inhibition. Front. Hum. Neurosci. 4 (2010).

42. P. L. Nunez, B. M. Wingeier, R. B. Silberstein, Spatial-temporal structures of human alpha rhythms: Theory, microcurrent sources, multiscale measurements, and global binding of local networks. Hum Brain Mapp 13, 125-164 (2001).

43. A. J. Quinn, D. Vidaurre, R. Abeysuriya, R. Becker, A. C. Nobre, M. W. Woolrich, TaskEvoked Dynamic Network Analysis Through Hidden Markov Modeling. Front. Neurosci. 12 (2018).

44. S. Suzuki, M. Grabowecky, Evidence for Perceptual "Trapping" and Adaptation in Multistable Binocular Rivalry. Neuron 36, 143-157 (2002).

45. C. L. E. Paffen, D. Alais, Attentional Modulation of Binocular Rivalry. Front Hum Neurosci 5 (2011).

46. C. L. E. Paffen, D. Alais, F. A. J. Verstraten, Attention speeds binocular rivalry. Psychol 
Sci 17, 752-756 (2006).

47. D. C. Van Essen, C. H. Anderson, D. J. Felleman, Information processing in the primate visual system: An integrated systems perspective. Science 255, 419 (1992).

48. K. Wunderlich, K. A. Schneider, S. Kastner, Neural correlates of binocular rivalry in the human lateral geniculate nucleus. Nat Neurosci 8, 1595-1602 (2005).

49. P. C. Klink, P. R. Roelfsema, Binocular rivalry outside the scope of awareness. Proceedings of the National Academy of Sciences 113, 8352-8354 (2016).

50. F. Tong, K. Nakayama, J. T. Vaughan, N. Kanwisher, Binocular Rivalry and Visual Awareness in Human Extrastriate Cortex. Neuron 21, 753-759 (1998).

51. L. Scocchia, M. Valsecchi, J. Triesch, Top-down influences on ambiguous perception: The role of stable and transient states of the observer. Front Hum Neurosci 8 (2014).

52. V. A. F. Lamme, H. Sup `er, R. Landman, P. R. Roelfsema, H. Spekreijse, The role of primary visual cortex (V1) in visual awareness. Vision Research 40, 1507-1521 (2000).

53. F. Tong, M. Meng, R. Blake, Neural bases of binocular rivalry. Trends in Cognitive Sciences $10,502-511(2006)$.

54. D. Carmel, V. Walsh, N. Lavie, G. Rees, Right parietal TMS shortens dominance durations in binocular rivalry. Current Biology 20, R799-R800 (2010).

55. R. Kanai, B. Bahrami, G. Rees, Human Parietal Cortex Structure Predicts Individual Differences in Perceptual Rivalry. Current Biology 20, 1626-1630 (2010).

56. J. C. Wilcke, R. P. O’Shea, R. Watts, Frontoparietal activity and its structural connectivity in binocular rivalry. Brain Research 1305, 96-107 (2009).

57. S. Fr“assle, J. Sommer, A. Jansen, M. Naber, W. Einh“auser, Binocular Rivalry: Frontal Activity Relates to Introspection and Action But Not to Perception. J. Neurosci. 34, 1738- 
1747 (2014).

58. T. Knapen, J. Brascamp, J. Pearson, R. van Ee, R. Blake, The role of frontal and parietal brain areas in bistable perception. J. Neurosci. 31, 10293-10301 (2011).

59. C. Koch, N. Tsuchiya, Attention and consciousness: Two distinct brain processes. Trends Cogn. Sci. (Regul. Ed.) 11, 16-22 (2007).

60. P. Fries, Rhythms For Cognition: Communication Through Coherence. Neuron 88, 220 235 (2015).

61. M. Meng, F. Tong, Can attention selectively bias bistable perception? Differences between binocular rivalry and ambiguous figures. Journal of Vision 4, 2-2 (2004).

62. L. Scocchia, M. Valsecchi, K. R. Gegenfurtner, J. Triesch, Differential effects of visual attention and working memory on binocular rivalry. Journal of Vision 14, 13-13 (2014). 63. B. Chen, Z. Zhu, R. Na, W. Fang, W. Zhang, Q. Zhou, S. Zhou, H. Lei, A. Huang, T. Chen, D. Ni, Y. Gu, J. Liu, F. Fang, Y. Rao, Genomic Analyses of Visual Cognition: Perceptual Rivalry and Top-Down Control. J. Neurosci. 38, 9668-9678 (2018).

64. S. M. Miller, N. K. Hansell, T. T. Ngo, G. B. Liu, J. D. Pettigrew, N. G. Martin, M. J. Wright, Genetic contribution to individual variation in binocular rivalry rate. Proc. Natl. Acad. Sci. U.S.A. 107, 2664-2668 (2010).

65. A. E. Cavanna, M. R. Trimble, The precuneus: A review of its functional anatomy and behavioural correlates. Brain 129, 564-583 (2006).

66. D. P. Carmel, E. Freeman, N. Lavie, G. Rees, Working memory maintains perceptual biases during binocular rivalry. Journal of Vision 4, 246-246 (2004).

67. C. M. Michel, T. Koenig, EEG microstates as a tool for studying the temporal dynamics of whole-brain neuronal networks: A review. NeuroImage 180, 577-593 (2018). 
68. C. G. Davey, J. Pujol, B. J. Harrison, Mapping the self in the brain's default mode network.

NeuroImage 132, 390-397 (2016).

69. Z. Huang, J. Zhang, J. Wu, G. A. Mashour, A. G. Hudetz, Temporal circuit of macroscale dynamic brain activity supports human consciousness. Science Advances 6, eaaz0087

(2020).

\section{Acknowledgments}

The authors declare that they have no competing interests or funding in support for this research. All data needed to evaluate the conclusions in the paper are publicly available in the repository: (https://datadryad.org/stash/dataset/doi:10.5061/dryad.bf1b1). Acknowledgements are given to the research team led by Bin He from the Department of Biomedical Engineering and Department of Psychology in the University of Minnesota for data collection and deposition; and Abhrajeet V. Roy for the correspondence regarding to data clarification. 


\section{Supplementary Files}

This is a list of supplementary files associated with this preprint. Click to download.

- BinocularRivalrySI2.pdf

- NCOMMS2124200rs.pdf 